\title{
Drug-Induced Hospital-Acquired Acute Kidney Injury in China: A Multicenter Cross-Sectional Survey
}

\author{
Chen Liu ${ }^{a}$ Suying Yan ${ }^{a}$ Yuqin Wang ${ }^{a}$ Jinwei Wang ${ }^{b}$ Xiujuan Fu \\ Hongtao Song $^{d}$ Rongsheng Tong $^{\mathrm{e}}$ Mei Dong $^{\mathrm{f}}$ Weihong Ge $^{\mathrm{g}}$ Jiawei Wang ${ }^{\mathrm{h}}$ \\ Hui Yang ${ }^{i}$ Changlian Wang $^{j}$ Peiyuan Xiak Limei Zhaol Sijing Shen ${ }^{\mathrm{m}}$ \\ Juan Xie ${ }^{n}$ Yangui Xu ${ }^{\circ}$ Peizhi Ma ${ }^{p}$ Hongjian Li ${ }^{q}$ Shegui Lu $^{r}$ Yufeng Ding ${ }^{\mathrm{s}}$ \\ Ling Jiang ${ }^{t}$ Yang Lin $^{\mathrm{u}}$ Maoyi Wang ${ }^{v}$ Feng Qiu $^{w}$ Wanyu Feng $^{\mathrm{x}}$ Li Yang $^{\mathrm{b}}$
}

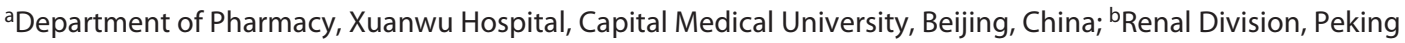
University First Hospital, Peking University, Beijing, China; 'Department of Pharmacy, The Second Hospital of Jilin University, Changchun, China; 'Department of Pharmacy, 900 Hospital of the Joint Logistic Team, Fuzhou, China; eDepartment of Pharmacy, Sichuan Provincial People's Hospital, Chengdu, China; fDepartment of Pharmacy, Affiliated Tumor Hospital Harbin Medical University, Harbin, China; ${ }^{9}$ Department of Pharmacy, Nanjing Drum

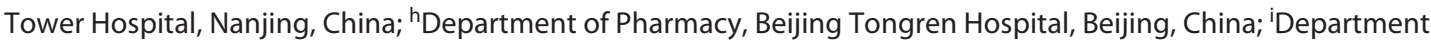
of Pharmacy, Qinghai University Affiliated Hospital, Xining, China; 'Department of Pharmacy, The First Affiliated

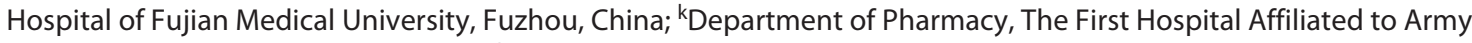
Medical University, Chongqing, China; 'Department of Pharmacy, Shengjing Hospital of China Medical University, Shenyang, China; ${ }^{m}$ Department of Pharmacy, Peking University Shougang Hospital, Beijing, China; ${ }^{\mathrm{n} D e p a r t m e n t}$ of Pharmacy, Guizhou Provincial People's Hospital, Guiyang, China; 'Department of Pharmacy, Tianjin First

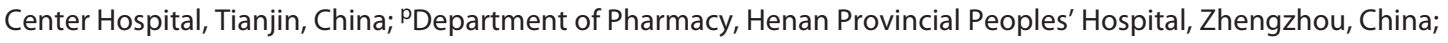
qDepartment of Pharmacy, Shandong Provincial Qianfoshan Hospital, Jinan, China; 'Department of Pharmacy, The Second Hospital of Nanchang University, Nanchang, China; ' Department of Pharmacy, The Tongji Affiliated Hospital of Tongji Medical College, Huazhong University of science, Wuhan, China; 'Department of Pharmacy, Anhui Provincial Hospital, Hefei, China; " Department of Pharmacy, Beijing Anzhen Hospital, Beijing, China; ${ }^{\vee}$ Department of Pharmacy, First Affiliated Hospital of Xi'an Jiaotong University, Xi'an, China; 'Department of Pharmacy, First Affiliated Hospital of Chongqing Medical University, Chongqing, China; ${ }^{x}$ Department of Pharmacy, Peking University People's Hospital, Beijing, China

\section{Keywords}

Drug-induced acute kidney injury · Hospital-acquired acute kidney injury

Chen Liu and Suying Yan contributed equally to this work and should be considered co-first authors.

karger@karger.com www.karger.com/kdd

Karger $\stackrel{\text { ' }}{5}$ BOPEN ACCESS
(C) 2020 The Author(s)

Published by S. Karger AG, Basel

This article is licensed under the Creative Commons AttributionNonCommercial-NoDerivatives 4.0 International License (CC BYNC-ND) (http://www.karger.com/Services/OpenAccessLicense) Usage and distribution for commercial purposes as well as any distribution of modified material requires written permission.

\section{Abstract}

Introduction: Drug-induced acute kidney injury (D-AKI) is one of the important types of AKI. The incidence of D-AKI in China has rarely been studied. Objective: This study aims to explore the disease burden, related drugs, and risk factors of

\section{Li Yang}

Renal Division

Peking University First Hospital, Peking University

No. 8 Xishiku Street, Xicheng District, Beijing 100034 (China)

li.yang@bjmu.edu.cn

Yuqin Wang

Department of Pharmacy, Xuanwu Hospital, Capital Medical University

No. 45 Changchun Street, Xicheng District

Beijing 100053 (China)

18601061925@163.com 
D-AKI. Methods: A nationwide cross-sectional survey was conducted in adult patients from 23 academic hospitals in 17 provinces in China. Suspected AKI was screened based on serum creatinine changes in accordance with the 2012 Kidney Disease: Improving Global Outcomes Clinical Practice Guideline for AKI, patients who met the diagnosis of hospital-acquired AKI in January and July of 2014 were defined. Suspected AKI was firstly evaluated for the possibility of DAKI by pharmacists using the Naranjo Scale and finally defined as D-AKI by nephrologists through reviewing AKI clinical features. Results: Altogether 280,255 hospitalized patients were screened and 1,960 cases were diagnosed as hospital-acquired AKI, among which 735 cases were defined as having D-AKI $(37.50 \%, 735 / 1,960)$ with an in-hospital mortality rate of $13.88 \%$ and $54.34 \%$ of the survivors did not achieve full renal recovery. 1,642 drugs were related to AKI in these patients. Anti-infectives, diuretics, and proton pump inhibitors were the top 3 types of drugs relevant to D-AKI, accounting for $66.63 \%$ cumulatively. Besides age, AKI staging, severe disease, hypoalbuminemia, plasma substitute, and carbapenem related D-AKI were independent risk factors for in-hospital mortality of D-AKI patients. Conclusion: In China, D-AKI has caused a substantial medical burden. Efforts should be made to pursue nephrotoxic drug stewardship to minimize attributable risk and improve the prevention, diagnosis, and treatment of D-AKI.

(C) 2020 The Author(s)

Published by S. Karger AG, Basel

\section{Introduction}

Drug-induced acute kidney injury (D-AKI) is an important type of AKI. It can be caused by various medications with diverse clinical manifestations and involving multiple clinical disciplines. In 2017, Awdishu and Mehta proposed the 6 R's of identifying and managing D-AKI, that is, Risk, Recognition, Response, Renal Support, Rehabilitation, and Research [1], providing a comprehensive and systematic strategy for the prevention and management of D-AKI. However, reports from different countries have shown that the characteristics of D-AKI have obvious regional difference. Therefore, it is necessary for nephrologists and pharmacists to fully understand the types of the disease-related drugs, medication habits, and status of clinical diagnosis and treatment in local areas.

At present, there is a lack of national data on the occurrence of D-AKI in China. A few existing studies (mainly single-center reports) showed that in the past 10 years, the percentage of D-AKI in all AKI increased from
26.5 to $42.9 \%$ [2-4]. In addition, the types of drugs that cause AKI reported in different cities vary, bringing great challenges to the development of national D-AKI prevention strategies. In this study, we investigated D-AKI occurrence in adult hospitalized patients from 23 academic hospitals in 17 provinces, which provided reliable data for the incidence of D-AKI in China. The spectrum of suspicious drugs, clinical characteristics, and the diagnosis and treatment status of D-AKI patients was reported. Subgroup analysis based on the administrative division and economic development was performed to provide important information for developing regional prevention and control strategies.

\section{Materials and Methods}

\section{Study Design and Study Objects}

We conducted a cross-sectional survey in mainland China, which included 17 provinces, autonomous regions, and municipalities (Appendix 1) with at least 1 teaching hospital in each capital city being selected. The survey was conducted in 3 steps. The main investigation methods refer to a previously published nationwide survey of AKI in China [5]. First, based on changes in serum creatinine ( $\mathrm{SCr}$ ), adult inpatients ( $\geq 18$ years old) in the hospital laboratory information system during 2014 were screened to identify "suspected AKI" in accordance with the AKI SCr criteria of 2012 Kidney Disease: Improving Global Outcomes (KDIGO) Clinical Practice Guideline for Acute Kidney Injury [6]. Second, the medical records of "suspected AKI" patients who were discharged from the hospitals in the 2 months of January and July 2014 were reviewed to confirm the diagnosis of AKI. The exclusion criteria included the following: patients with stage 5 chronic kidney disease (CKD); increase in SCr during hospitalization due to kidney resection; peak SCr value during hospitalization lower than $60 \mu \mathrm{mol} / \mathrm{L}$; other changes in SCr not explained by AKI. Community-acquired AKI was not the target in this study; therefore, patients diagnosed with AKI at admission were excluded. Patients with a hospital stay of 90 days and longer and patients with incomplete medical records were also excluded. Third, the Naranjo Adverse Drug Reaction Probability Scale (Naranjo Scale; see online suppl. Table 1; see www.karger.com/doi/10.1159/000510455 for all online suppl. material) [7] was used by the pharmacologist team to screen AKI cases that could be related to drugs, that is, suspected $\mathrm{D}$-AKI cases, and then the nephrologist team reviewed the medical record of each suspected D-AKI case to determine the rationale of $\mathrm{D}$-AKI diagnosis based on evaluating the clinical features of kidney injury and its relationship with the potential causal medications. If a patient had multiple factors that could be attributed to the AKI development, D-AKI would not be identified for the uncertainty of the diagnosis. If there were $>1$ drug that could be associated with AKI development after nephrologist evaluation, all the potential causal drugs would be recorded in this case.

We collected clinical data of confirmed D-AKI patients, including sociodemographic characteristics, comorbidities, inpatient departments, severe disease conditions, highest AKI stage [6], renal replacement therapy, hospitalization days, hospitalization costs 
and medication costs, concomitant nephrotoxic medication, hospital all-cause mortality, and AKI renal recovery. Severe disease conditions were defined as having any of the following disease conditions, including sepsis, shock, and multiple organ failure.

\section{Definitions}

We defined renal recovery at discharge as full recovery with SCr levels below the threshold or baseline level, partial recovery as a decrease in SCr of $25 \%$ or more from the peak concentration but remaining higher than the threshold or baseline level, and failure to recover as dependent on dialysis or a decrease in SCr of $<25 \%$ from the peak concentration [5]. The baseline SCr level was defined as the lowest SCr value that was available within 3 months prior to admission and throughout the hospital stay. For patients who had no reliable SCr record before admission and no evidence of baseline CKD, a back-estimation of the baseline SCr level was performed based on the 4-variable MDRD (Modification of Diet in Renal Disease) Study equation with the assumption of an estimated glomerular filtration rate (eGFR) of $75 \mathrm{~mL} / \mathrm{min} / 1.73 \mathrm{~m}^{2}$, following the recommendations of the 2012 KDIGO AKI clinical practice guideline $[6,8]$.

\section{Quality Control}

Investigators in each center included at least 2 pharmacists and 1 senior nephrologist, and all had received complete research training prior to this study. To standardize and control the homogeneity of the study, $\approx 5 \%$ of the cases from each center were randomly selected to check the validity of the questionnaires according to the electronic medical records. Repeated training was provided to the investigators when the questionnaire validity rate was below $90 \%$.

\section{Statistical Analysis}

The percentage of D-AKI was determined as the proportion of confirmed D-AKI cases out of the total hospital-acquired AKI patients. The number of D-AKI medications was the number of drugs that were defined associated with D-AKI development. The D-AKI-related drug percentage was the proportion of drugs used out of the total number of drugs associated with D-AKI. The percentage of D-AKI was calculated and compared among different regions and different economic development levels. GDP per capita data (PCGDP) published by the National Health and Family Planning Commission and National Bureau of Statistics of the People's Republic of China 2014 Statistics [9] were used to evaluate the level of economic development in different regions.

Continuous data were presented as the mean \pm SD or median (IQR) as appropriate and categorical variables as $n(\%)$. Clinical manifestation of patients stratified by geographical region and PCGDP were described. We compared groups using one-way ANOVA (if normally distributed) or the Kruskal-Wallis test (if in skewed distribution) for continuous variables and the $\chi^{2}$ test for categorical variables.

We analyzed relevant covariates that might associate with allcause in-hospital death of D-AKI patients (yes vs. no) with multivariate logistic regression and reported $p$ values from the Wald $\chi^{2}$ test and odds ratios (95\% confidence interval [CI]). Covariates included in the analysis were age (change by 10 years), sex (male vs. female), AKI stage at peak, PCGDP group, cardiovascular disease (yes or no), diabetes (yes or no), severe diseases (yes or no), cardiac surgery (yes or no), eGFR at hospitalization lower than $60 \mathrm{~mL} /$ $\mathrm{min} / 1.73 \mathrm{~m}^{2}$ (yes or no), hypoalbuminemia (serum albumin concentration lower than $35 \mathrm{~g} / \mathrm{L}$, yes or no), $>3$ nephrotoxic medications (yes or no), and different nephrotoxic medication types (yes or no). Relevant covariates that might be associated with AKI recovery in surviving D-AKI patients (yes or no) were also analyzed with multivariate logistic regression.

Microsoft Office Access 2013 software (Seattle, WA, USA) was used for data entry and management. Analyses were performed with IBM SPSS Statistics 19 software (Armonk, NY, USA). All $p$ values are 2 -sided, and a $p$ value of $<0.05$ was deemed significant.

\section{Results}

\section{Disease Burden of D-AKI}

280,255 adult hospitalized patients who were discharged in January and July of 2014 from 23 academic hospitals in 17 provinces were screened, and 3,564 patients with "suspected AKI" were identified by using KDIGO criteria. 1,960 patients were confirmed as having hospital-acquired AKI, among which 735 cases were identified with D-AKI (37.50\%, 735/1,960; online suppl. Table 2; Fig. 1). There was no significant regional difference in the proportion of D-AKI out of the total hospitalacquired AKI, which accounted for 37.56\% (418/1,113), $37.23 \%$ (191/513), and 37.72\% (126/334) in Northern, Southern, and Southwest China, respectively $(p=0.99$; online suppl. Table 2). The 23 hospitals were divided into high-, medium-, and low-PCGDP groups, and the proportion of D-AKI was significantly higher in regions with a lower PCGDP than that in medium- and high-PCGDP regions: $40.81 \%(242 / 593)$ versus $39.35 \%$ (305/775) versus $31.76 \%(188 / 592)(p=0.002)$.

The in-hospital mortality of D-AKI was $13.88 \%$ (102/735). Of the 633 survivors, 45.66\% (289/633) had full renal recovery, $19.43 \%$ (123/633) had partial recovery, and $34.91 \%(221 / 633)$ failed to recover renal function. The average length of hospital stay was $21.67 \pm 15.04$ days. The total hospitalization cost was US $\$ 8,076(3,848$, $14,825)$, and the medication cost was US $\$ 2,909(1,331$, 5,688) (Table 1).

\section{Clinical Features of D-AKI Patients}

Among the $735 \mathrm{D}$-AKI patients, 70 were treated in the intensive care unit (ICU) $(9.52 \%), 330$ in the internal medicine department (44.90\%), and 335 in the surgery (45.58\%) department. Patients were $62 \pm 17$ years old with $64.90 \%$ (477/735) of males. Comorbidities included diabetes $(189 / 735,25.71 \%)$, cardiovascular disease (398/735, 54.15\%), CKD (255/735, 34.69\%), and hypoalbuminemia $(170 / 735,23.13 \%)$. AKI staging was defined 
Table 1. Characteristics of patients with D-AKI in different regions

\begin{tabular}{|c|c|c|c|c|c|}
\hline & $\begin{array}{l}\text { All } \\
(n=735)\end{array}$ & $\begin{array}{l}\text { North } \\
(n=418)\end{array}$ & $\begin{array}{l}\text { South } \\
(n=191)\end{array}$ & $\begin{array}{l}\text { Southwest } \\
(n=126)\end{array}$ & $\begin{array}{l}p \\
\text { value }\end{array}$ \\
\hline \multicolumn{6}{|l|}{ Ward, $n(\%)$} \\
\hline ICU & $70(9.52)$ & $39(9.33)$ & $25(13.09)$ & $6(4.76)$ & 0.14 \\
\hline Medicinal & $330(44.90)$ & $185(44.26)$ & $81(42.41)$ & $64(50.79)$ & \\
\hline Surgical & $335(45.58)$ & $194(46.41)$ & $85(44.50)$ & $56(44.44)$ & \\
\hline Male, $n(\%)$ & $477(64.90)$ & $258(61.72)$ & $130(68.06)$ & $89(70.63)$ & 0.11 \\
\hline Age, years & $62(17)$ & $63(16)$ & $62(17)$ & $60(18)$ & 0.31 \\
\hline \multicolumn{6}{|l|}{ Age group, $n(\%)$} \\
\hline Aged $18-39$ years & $73(9.93)$ & $37(8.85)$ & $21(10.99)$ & $15(11.90)$ & 0.94 \\
\hline Aged $40-59$ years & $234(31.84)$ & $133(31.82)$ & $60(31.41)$ & $41(32.54)$ & \\
\hline Aged $60-79$ years & $312(42.45)$ & $182(43.54)$ & $78(40.84)$ & $52(41.27)$ & \\
\hline Aged $\geq 80$ years & $116(15.78)$ & $66(15.79)$ & $32(16.75)$ & $18(14.29)$ & \\
\hline Baseline eGFR, $\mathrm{mL} / \mathrm{min} / 1.73 \mathrm{~m}^{2}$ & $85(56,103)$ & $86(57,104)$ & $80(50,100)$ & $84(55,104)$ & 0.13 \\
\hline \multicolumn{6}{|l|}{ Comorbidity, $n(\%)$} \\
\hline Diabetes & $189(25.71)$ & $110(26.32)$ & $37(19.37)$ & $42(33.33)$ & 0.02 \\
\hline CVD & $398(54.15)$ & $233(55.74)$ & $104(54.45)$ & $61(48.41)$ & 0.35 \\
\hline Pre-existing CKD & $255(34.69)$ & $136(32.54)$ & $72(37.70)$ & $47(37.30)$ & 0.37 \\
\hline Severe diseases & $72(9.80)$ & $42(10.05)$ & $17(8.90)$ & $13(12.64)$ & 0.89 \\
\hline Hypoalbuminemia & $170(23.13)$ & $103(24.64)$ & $29(15.18)$ & $38(30.16)$ & 0.004 \\
\hline \multicolumn{6}{|l|}{ AKI-related drugs, $n(\%)$} \\
\hline 1 & $302(41.09)$ & $186(44.50)$ & $65(34.03)$ & $51(40.48)$ & 0.007 \\
\hline $2-3$ & $308(41.90)$ & $183(43.78)$ & $83(38.22)$ & $52(43.27)$ & \\
\hline$>3$ & $125(17.01)$ & $49(11.72)$ & $43(27.75)$ & $23(16.25)$ & \\
\hline \multicolumn{6}{|l|}{ AKI stage, $n(\%)$} \\
\hline AKI-1 & $532(72.38)$ & $317(75.84)$ & $129(67.54)$ & $86(68.25)$ & 0.10 \\
\hline AKI-2 & $123(16.73)$ & $66(15.79)$ & $35(18.32)$ & $22(17.46)$ & \\
\hline AKI-3 & $80(10.88)$ & $35(8.37)$ & $27(14.14)$ & $18(14.29)$ & \\
\hline Peak SCr, $\mu \mathrm{mol} / \mathrm{L}$ & $146(99,226)$ & $133(94,211)$ & $162(109,252)$ & $157(110,251)$ & $<0.001$ \\
\hline Mortality, $n(\%)$ & $102(13.88)$ & $69(16.51)$ & $14(7.33)$ & $19(15.08)$ & 0.009 \\
\hline \multicolumn{6}{|l|}{ Renal recovery, ${ }^{\mathrm{a}} n(\%)$} \\
\hline Full recovery & $289(45.66)$ & $175(50.14)$ & $68(38.42)$ & $46(42.99)$ & 0.03 \\
\hline Partial recovery & $123(19.43)$ & $60(17.19)$ & $46(25.99)$ & $17(15.89)$ & \\
\hline Failure to recover & $221(34.91)$ & $114(32.66)$ & $63(35.59)$ & $44(41.12)$ & \\
\hline Hospital stay, days & $22(15)$ & $21(15)$ & $23(15)$ & $21(16)$ & 0.30 \\
\hline Hospital cost $(U S \$)^{\mathrm{b}}$ & $8,076(3,848,14,825)$ & $8,059(3,766,14,492)$ & $9,033(4,310,17,411)$ & $5,322(3,399,13,676)$ & $<0.001$ \\
\hline Medication cost (US\$) & $2,909(1,331,5,688)$ & $2,784(1,324,5,582)$ & $3,435(1,582,6,348)$ & $2,206(1,185,5,307)$ & 0.006 \\
\hline
\end{tabular}

Data are mean (SD), $n$ (\%), or median (IQR), unless stated otherwise. D-AKI, drug-induced acute kidney injury; ICU, intensive care unit; eGFR, estimated glomerular filtration rate; CVD, cardiovascular disease; CKD, chronic kidney disease; SCr, serum creatinine. ${ }^{a}$ Renal recovery only includes patients who were alive when discharge. ${ }^{b}$ Data missing for hospital cost in 40 cases ( 9 in the south vs. 31 in the southwest) and for medication cost in 23 cases ( 9 in the south vs. 14 in the southwest).

as stage 1 in $532(72.38 \%)$, stage 2 in $123(16.73 \%)$, and stage 3 in $80(10.88 \%)$ patients (Table 1$)$.

A total of 1,642 drugs were related to kidney injury. DAKI was relevant to a single drug in $41.09 \%(302 / 735)$, to $2-3$ drugs in $41.90 \%$ (308/735), and to $>3$ drugs in $17.01 \%$ $(125 / 735)$ of patients (Table 1$)$. With the increase in the number of AKI-related drugs, the value of peak SCr tended to increase ( $p=0.06$, Fig. 2). Furthermore, there were more cases in Southern China where D-AKI was caused by $>3$ drugs compared with those in Northern China and Southwest China ( 27.75 vs. 11.72 vs. $16.25 \%$; $p=0.007$ ), suggesting a potential more complicated medication prescription in Southern China.

\section{Types of Drugs Relevant to Kidney Injury in D-AKI \\ Patients}

The most common types of drugs that were defined relevant to kidney injury included anti-infectives (34.35\%, 


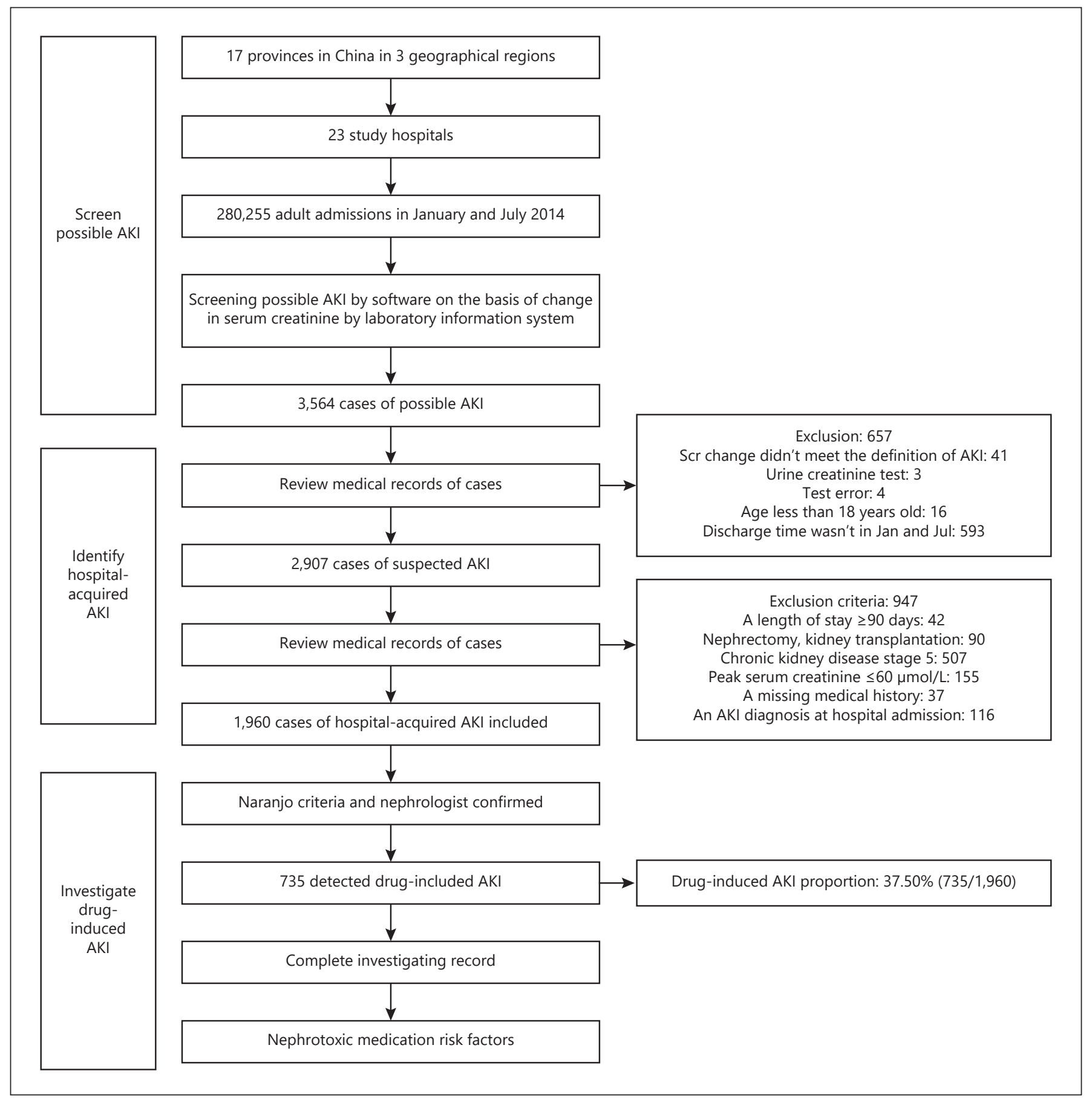

Fig. 1. Flowchart of the total research process. AKI, acute kidney injury; D-AKI, drug-induced acute kidney injury; SCr, serum creatinine.

$564 / 1,642)$, diuretics $(21.80 \%, 358 / 1,642)$, and proton pump inhibitors (PPIs) $(10.48 \%, 172 / 1,642)$, followed by contrast agents $(4.57 \%, 75 / 1,642)$, nonsteroidal anti-inflammatory drugs (NSAIDs) $(4.08 \%, 67 / 1,642)$, renin- angiotensin system inhibitors (RASIs) $(4.02 \%, 66 / 1,642)$, anticoagulant/antiplatelet drugs $(3.96 \%, 65 / 1,642)$, antitumor drugs $(3.17 \%, 52 / 1,642)$, and neuropsychiatric drugs $(3.05 \%, 50 / 1,642)$ (Table 2). Cephalosporins, glyco- 
peptides, carbapenems, quinolones, and penicillins accounted for $86.70 \%$ of the total anti-infectives. Furosemide, mannitol, and torsemide accounted for $77.93 \%$ of the total diuretics. Pantoprazole, omeprazole, and esomeprazole accounted for $86.63 \%$ of the total PPIs (online suppl. Table 3).
We further analyzed D-AKI-related drugs in different regions. As shown in Table 2, anti-infectives and diuretics were the most common types of D-AKI-related drugs in the Northern, Southern, and Southwest regions. PPIs were also common AKI-related drugs in Southwest China and Northern China. D-AKI caused by

Table 2. Top 10 nephrotoxic medication types associated with D-AKI

\begin{tabular}{|c|c|c|c|c|c|}
\hline Medication type & $\begin{array}{l}\text { Total } \\
(n=1,642)\end{array}$ & $\begin{array}{l}\text { North } \\
(n=857)\end{array}$ & $\begin{array}{l}\text { South } \\
(n=501)\end{array}$ & $\begin{array}{l}\text { Southwest } \\
(n=284)\end{array}$ & $p$ value \\
\hline Anti-infective agents, $n(\%)$ & $564(34.35)$ & $297(34.66)$ & $177(35.33)$ & $90(31.69)$ & 0.57 \\
\hline PPIs, $n(\%)$ & $172(10.48)$ & $101(11.79)$ & $20(3.99)$ & $51(17.96)$ & $<0.001$ \\
\hline Contrast agents, $n(\%)$ & $75(4.57)$ & $57(6.65)$ & $17(3.39)$ & $1(0.35)$ & $<0.001$ \\
\hline NSAIDs, $n(\%)$ & $67(4.08)$ & $22(2.57)$ & $36(7.19)$ & $9(3.17)$ & $<0.001$ \\
\hline Anticoagulant/antiplatelet agents, $n(\%)$ & $65(3.96)$ & $28(3.27)$ & $21(4.19)$ & $16(5.63)$ & 0.20 \\
\hline Antitumor agents, $n(\%)$ & $52(3.17)$ & $25(2.92)$ & $22(4.39)$ & $5(1.76)$ & 0.11 \\
\hline Neuropsychiatric agents, $n(\%)$ & $50(3.05)$ & $26(3.03)$ & $17(3.39)$ & $7(2.46)$ & 0.77 \\
\hline Plasma substitutes, $n(\%)$ & $47(2.86)$ & $38(4.43)$ & $3(0.60)$ & $6(2.11)$ & $<0.001$ \\
\hline Others, $n(\%)$ & $126(7.67)$ & $53(6.18)$ & $50(9.98)$ & $23(8.10)$ & 0.04 \\
\hline
\end{tabular}

Data are \%, unless stated otherwise. D-AKI, drug-induced acute kidney injury; PPIs, proton pump inhibitors; NSAIDs, nonsteroidal anti-inflammatory drugs; ACEIs, angiotensin converting enzyme inhibitors; ARBs, angiotensin receptor blockers.

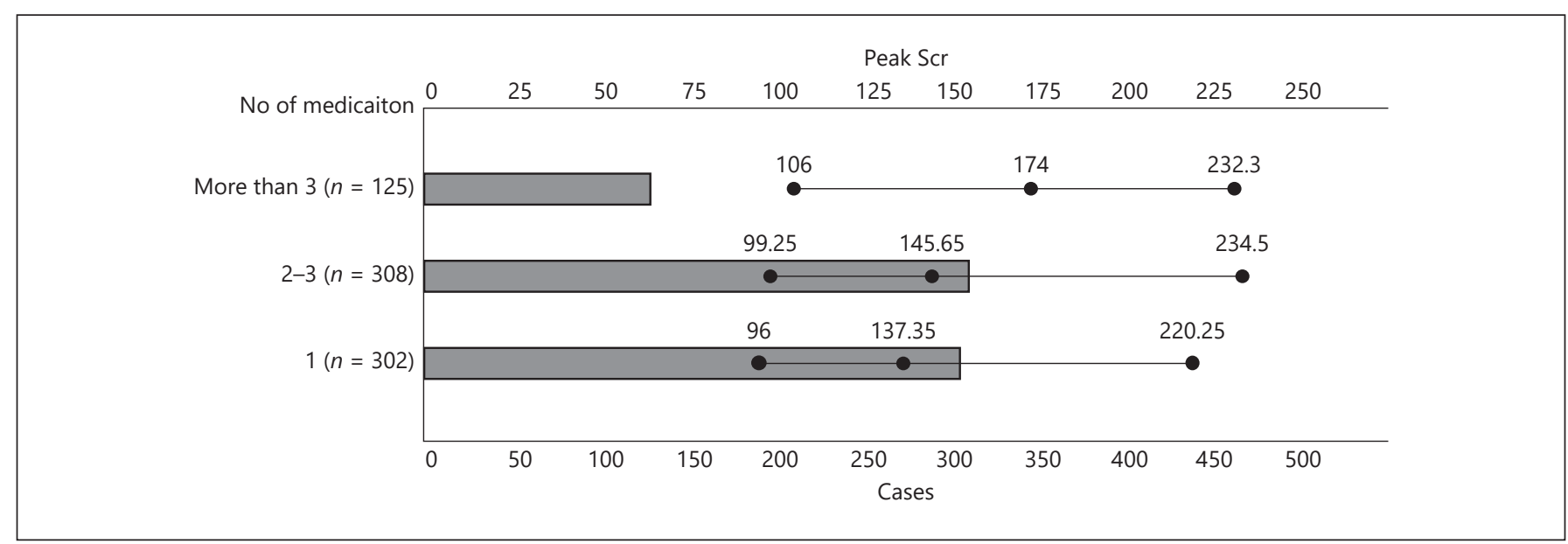

Fig. 2. With the increase in the number of AKI-related drugs used, the SCr peak value increased, but the difference did not reach statistical significance $(p=0.06)$. AKI, acute kidney injury; SCr, serum creatinine.

Fig. 3. Different types of drugs were associated with various clinical features of D-AKI. The time interval between drug administration and AKI diagnosis was $3(2,6)$ days. Contrast agents, plasma substitutes, NSAIDs, and neuropsychiatric drugs caused the fastest AKI occurrence, that is, $2(1,6)$ days. The occurrence of AKI induced by anticoagulant and antiplatelet drugs was the slowest, that is, $5(2,8)$ days. Peak SCr levels were the highest in RASI-induced D-AKI $(202[165,275] \mu \mathrm{mol} / \mathrm{L})$ and the lowest in antitumor druginduced AKI $(114.3[89,159] \mu \mathrm{mol} / \mathrm{L})$ and NSAID-induced AKI $(117[97,160] \mu \mathrm{mol} / \mathrm{L})$. D-AKI, drug-induced acute kidney injury; SCr, serum creatinine; NSAIDs, nonsteroidal anti-inflammatory drugs; RASIs, renin-angiotensin system inhibitors.

(For figure see next page.) 


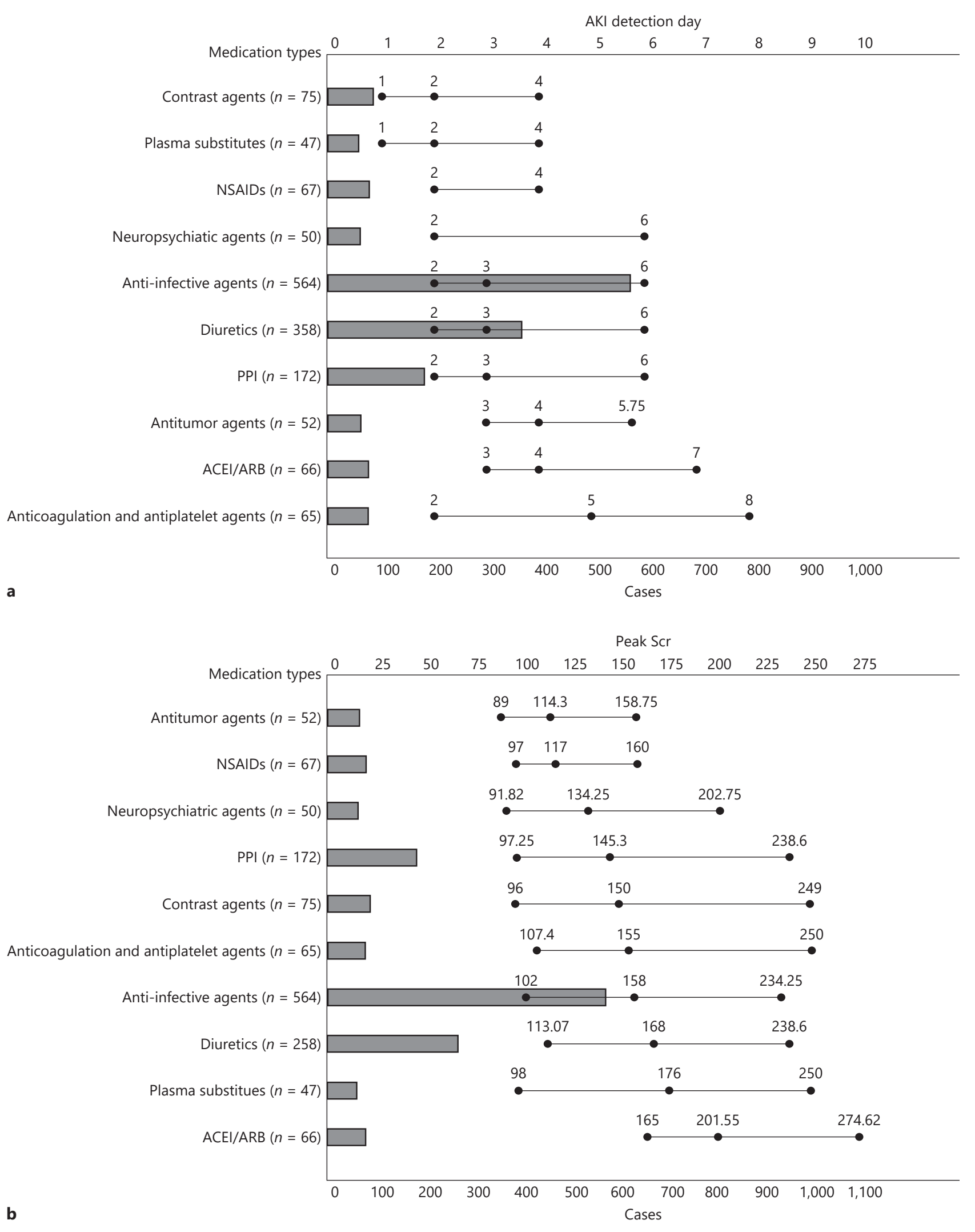


Mortality rate, \%

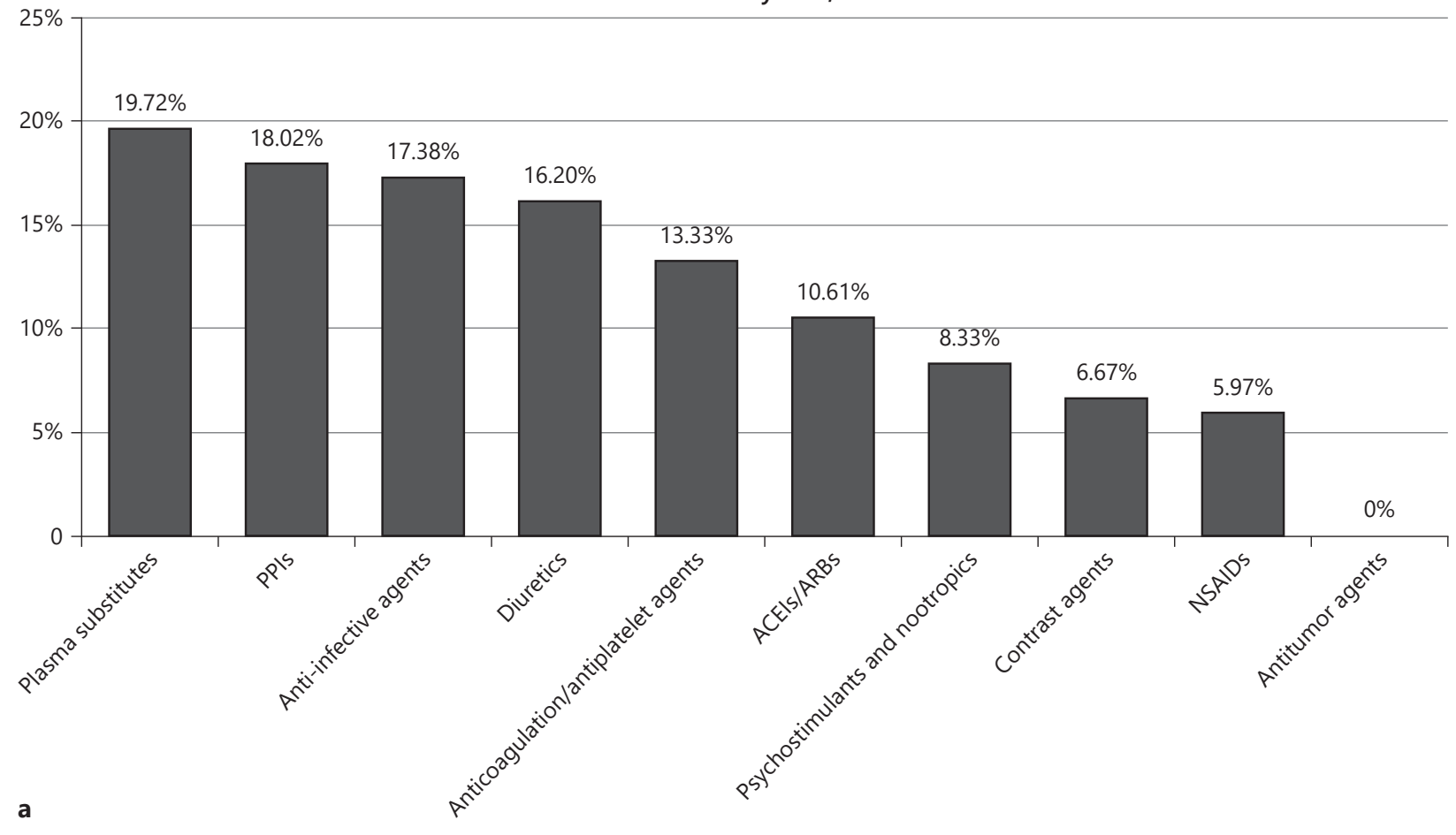

Renal recovery, \%

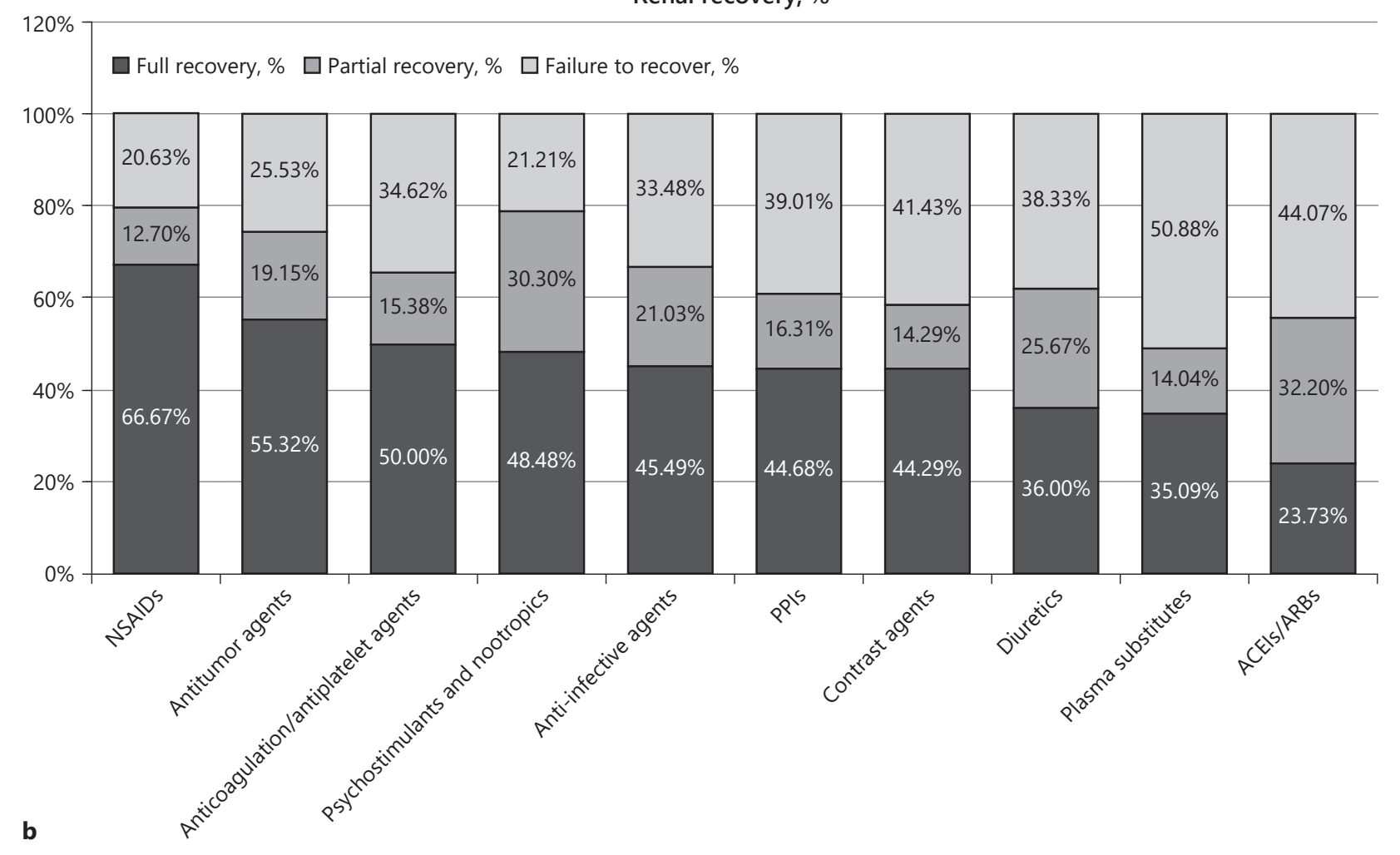

4

(For legend see next page.) 
NSAIDs and RASIs were relatively more common in Southern China. Contrast agent-induced D-AKI ranked fourth in the Northern region, ranked eighth in the Southern region, and was rare in the Southwest region. There was no significant difference in the types of drugs among regions according to the different levels of economic development (online suppl. Table 4). With respect to the specific medication, furosemide was the top drug associated with D-AKI in all regions and all medical departments, followed by vancomycin, pantoprazole, mannitol, torsemide, spironolactone, and omeprazole (online suppl. Tables 5, 6). Among the top $10 \mathrm{D}$ AKI-related drugs in different medical departments, anti-infectives accounted for the highest percentage in ICUs (50\%); diuretics were of the most common in internal medicine departments (40\%); and diuretics, antiinfectives, and PPIs accounted for $30 \%$ in surgery departments.

The aforementioned results suggested that in different regions and in different medical departments, because of the variety of disease profiles and the potential prescrip- tion propensity, the types of D-AKI-related drugs were different. It is necessary to fully understand the individual spectrum of D-AKI-related drugs in different regions to carry out effective clinical prevention and treatment (Fig. 3, 4).

\section{Analysis of Prognosis-Related Risk Factors}

The independent risk factors for in-hospital mortality included age (per 10 years of age) $(\mathrm{OR}=1.35,95 \% \mathrm{CI}$ : $1.13-1.61, p=0.001$ ), AKI stage (using stage 1 as the reference, stage 2 [OR $=3.81,95 \% \mathrm{CI}: 2.16-6.71, p<0.001]$; stage $3[\mathrm{OR}=3.64,95 \% \mathrm{CI}: 1.90-6.96, p<0.001])$, severe disease $(\mathrm{OR}=5.50,95 \% \mathrm{CI}: 3.02-10.03, p<0.001)$, hypoalbuminemia (OR $=1.99,95 \% \mathrm{CI}: 1.19-3.32, p=0.009)$, plasma substitute-induced D-AKI $(\mathrm{OR}=2.72,95 \% \mathrm{CI}$ : 1.16-6.37, $p=0.02$ ), and carbapenem-induced D-AKI $(\mathrm{OR}=2.61,95 \% \mathrm{CI}: 1.35-5.04, p=0.004)($ Table 3$) . \mathrm{Of}$ those who survived from AKI and had records regarding renal recovery at discharge, the independent risk factors for failure to recover included baseline eGFR $<60 \mathrm{~mL} /$ $\mathrm{min} / 1.73 \mathrm{~m}^{2}(\mathrm{OR}=2.07,95 \% \mathrm{CI}: 1.40-3.06, p<0.001)$,

Table 3. Risk factors associated with all-cause in-hospital death

\begin{tabular}{|c|c|c|c|c|c|c|}
\hline & OR & $95 \% \mathrm{CI}$ & $p$ value & OR & $95 \% \mathrm{CI}$ & $p$ value \\
\hline \multicolumn{7}{|l|}{ AKI stage } \\
\hline AKI stage 1 & $1^{\mathrm{a}}$ & & & $1^{\mathrm{a}}$ & & \\
\hline AKI stage 2 & 4.24 & $2.81-7.67$ & $<0.001$ & 3.81 & $2.16-6.71$ & $<0.001$ \\
\hline Hypoalbuminemia (yes vs. no) & 3.21 & $2.07-4.97$ & $<0.001$ & 1.99 & $1.19-3.32$ & 0.009 \\
\hline Plasma substitutes (yes vs. no) & 2.84 & $1.43-5.63$ & 0.003 & 2.72 & $1.16-6.37$ & 0.02 \\
\hline Carbopenems (yes vs. no) & 2.78 & $1.60-4.84$ & $<0.001$ & 2.61 & $1.35-5.04$ & 0.004 \\
\hline Cardiovascular diseases & 1.58 & $1.03-2.44$ & 0.04 & 0.83 & $0.49-1.43$ & 0.51 \\
\hline Glycopeptide (yes vs. no) & 2.63 & $1.55-4.49$ & $<0.001$ & 1.60 & $0.85-2.99$ & 0.14 \\
\hline
\end{tabular}

All variables listed in the table were included in the multivariate logistic regression model. AKI, acute kidney injury; GDP, gross domestic product; OR, odds ratio; CI, confidence interval. ${ }^{\text {a }}$ Reference value.

Fig. 4. Patients with D-AKI relevant to plasma substitutes had the highest in-hospital death rate (19.72\%) and the highest percentage of survivors failed to renal recover $(50.88 \%)$. Those who had DAKI associated with NSAIDs presented the lowest mortality
(5.97\%) and the lowest rate of kidney failure to recover (20.63\%). D-AKI, drug-induced acute kidney injury; NSAIDs, nonsteroidal anti-inflammatory drugs. 
Table 4. Risk factors associated with renal recovery

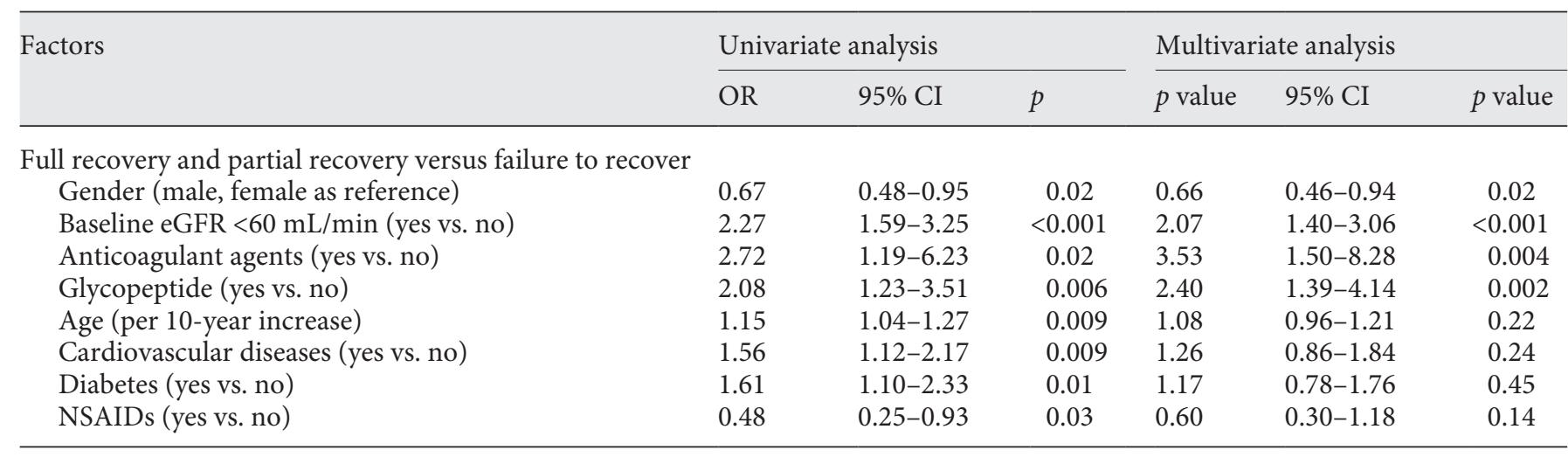

All variables listed in the table were included in the multivariate logistic regression model. eGFR, estimated glomerular filtration rate; NSAIDs, nonsteroidal anti-inflammatory drugs; OR, odds ratio; CI, confidence interval.

anticoagulant-induced D-AKI (OR $=3.53,95 \%$ CI: 1.50 $8.28, p=0.004)$, and glycopeptide-induced D-AKI (OR = 2.40, 95\% CI: 1.39-4.14, $p=0.002$ ) (Table 4).

\section{Discussion}

We carried out the first nationwide multicenter survey on the incidence of hospital-acquired D-AKI in China. This survey included 280,255 patients from 23 academic hospitals in 17 provinces. Patients with suspected AKI were electronically screened in accordance with KDIGO AKI SCr criteria, then evaluated for the possibility of DAKI by pharmacists using the Naranjo Scale, and finally defined as having D-AKI by nephrologists through reviewing AKI clinical features. By this way, we tried to avoid the possible overdiagnosis by using the Naranjo Scale alone and the missed diagnosis resulting from the ICD code review. According to our survey, D-AKI accounted for $37.50 \%$ of hospital-acquired AKI in China, with a hospital mortality rate of 13.88 and $54.34 \%$ of the survivors not achieving full renal recovery.

Globally, drugs are important causes of AKI. Existing research studies show that in developing countries, DAKI accounts for $21-37.4 \%$ of all AKI cases [10-13], which is higher than that in developed countries (19.4$27.4 \%)[14,15]$. In China, single-center studies have shown that the percentage of D-AKI out of total AKI has increased from 26.5 to $42.9 \%$ in the past 10 years [2-4]. Based on the multicenter data provided in this study, DAKI accounted for $37.50 \%$ of AKI in China, and the occurrence was related to regional economic development; the percentage of D-AKI in economically underdeveloped areas was $40.81 \%$, significantly higher than that in regions with a relatively developed economy (31.76\%). Therefore, D-AKI has already caused a serious disease burden in China, which is especially critical in economically underdeveloped areas.

The types of drugs that cause D-AKI are complex and have regional characteristics. According to a study based on the Food and Drug Administration Adverse Event Reporting System database, the drugs with high reporting odds ratios (RORs) for AKI in US included furosemide, vancomycin, spirolactam, lithium, and acyclovir [16]. Another recent study based on the Japanese Adverse Drug Event Report database indicated that the drugs with high RORs for AKI were valaciclovir, eldecalcitol, edaravone, acyclovir, piperacillin/tazobactam, and so on [17]. There is still a lack of national registration data for D-AKI in China. In the current study, we showed that furosemide, vancomycin, and pantoprazole are the top 3 drugs that cause D-AKI in China, while D-AKI caused by antiviral drugs is relatively rare. Although the potential renal toxicity of vancomycin is widely known, it is still the preferred and widely used drug for treating methicillin-resistant Staphylococcus aureus (MRSA) infection in China. According to a multicenter study that included 103 hospitals in 8 cities in China, there were 357,225 cases of vancomycin prescription in 2017 [18], and the incidence of vancomycin-induced AKI was $16.2 \%$ [19]. Although the study showed that $88.8 \%$ of the enrolled hospitals had the facility for vancomycin therapeutic drug monitoring (TDM) [19], 76.47\% of the patients were reported to have inadequate TDM [20] and the preassessment of the risk 
of kidney injury and renal function monitoring were generally lacking. Therefore, it is extremely urgent to improve vancomycin renal safety in China.

In recent years, the adverse effects of PPIs on the kidney have received increasing attention worldwide. Studies have shown that PPIs can increase the risk of AKI, acute interstitial nephritis (AIN), CKD, and end-stage renal disease $[21,22]$. PPIs (mainly omeprazole) were the most common drugs that cause drug-induced AIN in the elderly in the US $[23,24]$. The current study found that PPIs have become the third most common cause of DAKI in China, especially in the Northern and Southwest China. Among PPIs, pantoprazole is the most common in D-AKI, which may be related to the wide clinical application of this drug in China.

This investigation showed that different types of drugs caused different occurrence rates and severities of AKI, resulting in a complex clinical manifestation. For example, AKI caused by contrast agents and plasma substitutes was the fastest to manifest $(2[1,6]$ days), while AKI caused by anticoagulant and antiplatelet drugs was the slowest to manifest $(5[2,8]$ days). The clinical manifestations of D-AKI with a slow onset and relatively mild injury are clinically occult and can easily lead to delayed or missed diagnosis. D-AKI with a fast onset presents challenges for effective monitoring and timely treatment. To handle such a large number of drug types and complex clinical features of kidney injury, electronic surveillance of D-AKI could be the most powerful tool [1]. A good example is Nephrotoxic Injury Negated by Just-in-Time Action (NINJA) developed by the Stuart L. Goldstein group, which has been shown to successfully reduce AKI rates under nephrotoxic medication exposure [25].

Currently, there are few studies on the risk factors related to D-AKI patient outcomes. The large sample size of D-AKI cases in this study allowed us to analyze the common risk factors for D-AKI and further explore the effect of drugs on prognosis. We found that older age, severe disease conditions such as sepsis, shock, and multiple organ failure, advanced AKI stages, and hypoalbuminemia were independent risk factors for in-hospital mortality of patients with D-AKI, which were similar to those for AKI [3, 5, 26-29]. In addition, we found that plasma substitute-induced D-AKI and carbapenem-induced D-AKI were independent risk factors for the inhospital mortality; anticoagulant-induced D-AKI and glycopeptide-induced D-AKI were independent risk factors for failure to renal recovery. Other potential cofactors that were detected with high prevalence in the cur- rent cohort, for example, concomitant diseases such as diabetes, baseline CKD, and cardiovascular diseases, would also contribute to kidney failure to recover in DAKI patients. Our study suggests that for patients with D-AKI, long-term follow-up and management by nephrologists should be performed.

Our study has limitations related to the retrospective design. AKI was diagnosed based on the dynamic changes in SCr independently of urine output, which would have missed some AKI cases. The determination of DAKI was based on reviewing medical records and therefore might result in misdiagnosis in some cases. Patients who developed community-acquired AKI were excluded, which could impact nephrotoxic medication types. Because this study did not include individuals who did not develop AKI after medication use, it is impossible to discuss the risk factors for D-AKI development. In addition, since the information of hospitalization during the study period was not collected, we are not able to report the incidence of D-AKI in total hospital admission or in different departments. As most of the D-AKI cases detected in the current study were located in non-renal units, renal biopsy had not been performed in our cohort which limited our understanding about the various types of kidney injury induced by medications. Finally, because of the limitation of our data collection, we could not evaluate the roles of follow-up treatment in the prognosis of $\mathrm{D}$ AKI in our patients. The aforementioned limitations can only be overcome by prospective studies with establishment of an AKI warning system. In addition, since traditional Chinese medicine hospitals were not included in the study, we were not able to investigate D-AKI related to herbal medicines.

\section{Conclusion}

The prevention and treatment of D-AKI are challenging in China due to the large D-AKI population, diverse clinical features, and the complexity of drugs that cause kidney injury in different regions. Nephrologists and pharmacists should work together to formulate effective regional prevention and control strategies.

\section{Statement of Ethics}

This study was approved and patient written informed consent was waived by the Ethics Committee of Xuanwu Hospital, Capital Medical University, Beijing, China (No. [2015]011). 


\section{Conflict of Interest Statement}

The authors have no conflicts of interest to declare.

\section{Funding Sources}

This study was supported by the Medical Policy and Administration Department, National Health and Family Planning Commission of China, 2015; the National Natural Science Foundation of China (Nos. 91742205 and 81625004); the Beijing Young Scientist Program (BJJWZYJH01201910001006); and Peking University Clinical Scientist Program by the Fundamental Research Funds for the Central Universities.

\section{Author Contributions}

C.L. and S.Y. collected and analyzed the data, interpreted the results, and drafted the manuscript. L.Y. conceived and designed the study, interpreted the results, and revised the manuscript. L.Y. and Y.W. obtained funding, organized the study, and helped revise the manuscript. J.W. helped analyze the data. All authors contributed to data collection on site.

\section{Appendix 1}

List of Hospitals

Appendix 1a. List of Hospitals by Geographical Regions

- North: Beijing, Tianjin, Henan, Shandong, Heilongjiang, Jilin, Liaoning, Shanxi, and Qinghai.

Xuanwu Hospital Capital Medical University.

Peking University People's Hospital.

Beijing Anzhen Hospital.

Beijing Tongren Hospital.

Peking University Shougang Hospital.

Tianjin First Central Hospital.

Henan Provincial People's Hospital.

Shandong Provincial Qianfoshan Hospital.

Affiliated Tumor Hospital of Harbin Medical University.

The Second Hospital of Jilin University.

Shengjing Hospital of China Medical University.

First Affiliated Hospital of Xi'an Jiaotong University.
Qinghai University Affiliated Hospital.

- South: Fujian, Jiangxi, Anhui, Hubei, and Jiangsu.

The First Affiliated Hospital of Fujian Medical University. 900 Hospital of the Joint Logistic Team.

The Second Hospital of Nanchang University.

Anhui Provincial Hospital.

The Tongji Affiliated Hospital of Tongji Medical College, Huazhong University of Science.

Nanjing Drum Tower Hospital.

- Southwest: Guizhou, Chongqing, and Sichuan Guizhou Provincial People's Hospital.

First Affiliated Hospital of Chongqing Medical University. The First Hospital Affiliated to Army Medical University. Sichuan Provincial People's Hospital.

Appendix 1b. List of Hospitals by GDP per Capita

- High-GDP per capita group: Beijing, Tianjin, and Jiangsu. Xuanwu Hospital Capital Medical University.

Peking University People's Hospital.

Beijing Anzhen Hospital.

Beijing Tongren Hospital.

Peking University Shougang Hospital.

Tianjin First Central Hospital.

Nanjing Drum Tower Hospital.

- Medium-GDP per capita group: Shanxi, Hubei, Chongqing, Jilin, Fujian, Liaoning, and Shandong.

First Affiliated Hospital of Xi'an Jiaotong University.

The Tongji Affiliated Hospital of Tongji Medical College,

Huazhong University of Science.

The First Hospital Affiliated to Army Medical University.

First Affiliated Hospital of Chongqing Medical University.

The Second Hospital of Jilin University.

The First Affiliated Hospital of Fujian Medical University.

900 Hospital of the Joint Logistic Team.

Shengjing Hospital of China Medical University.

Shandong Provincial Qianfoshan Hospital.

- Low-GDP per capita group: Guizhou, Anhui, Jiangxi, Sichuan, Henan, Heilongjiang, and Qinghai.

Guizhou Provincial People's Hospital.

Anhui Provincial Hospital.

The Second Hospital of Nanchang University.

Sichuan Provincial People's Hospital.

Henan Provincial People's Hospital.

Affiliated Tumor Hospital of Harbin Medical University.

Qinghai University Affiliated Hospital.

\section{References}

1 Awdishu L, Mehta RL. The 6R's of drug induced nephrotoxicity. BMC Nephrol. 2017; 18(1):124-35.

2 Che ML, Yan YC, Zhang Y, Gu Y, Wang NS, Chen N, et al. [Analysis of drug-induced acute renal failure in Shanghai]. Zhonghua Yi Xue Za Zhi. 2009;89(11):744-9.

3 Xu X, Nie S, Liu Z, Chen C, Xu G, Zha Y, et al. Epidemiology and clinical correlates of AKI in Chinese hospitalized adults. Clin J Am Soc Nephrol. 2015;10(9):1510-8.
4 Wang Y, Cui Z, Fan M. Hospital-acquired and community-acquired acute renal failure in hospitalized Chinese: a ten-year review. Ren Fail. 2007;29(2):163-8.

5 Yang L, Xing G, Wang L, Wu Y, Li S, Xu G, et al. Acute kidney injury in China: a cross-sectional survey. Lancet. 2015;386(10002):1465-71.

6 Kidney Disease: Improving Global Outcomes (KDIGO) Acute Kidney Injury Work Group. KDIGO clinical practice guideline for acute kidney injury (2012). Kidney Int Suppl. 2012; 2:1-138.
7 Naranjo CA, Busto U, Sellers EM, Sandor P, Ruiz I, Roberts EA, et al. A method for estimating the probability of adverse drug reactions. Clin Pharmacol Ther. 1981;30(2):23945.

8 Wang Y, Wang J, Su T, Qu Z, Zhao M, Yang L. Community-acquired acute kidney injury: a nationwide survey in China. Am J Kidney Dis. 2017;69(5):647-57. 
9 National Health and Family Planning Commission. Statistics yearbook of health and family planning in China. Peking Union Medical College Press; 2015.

10 Jha V, Malhotra HS, Sakhuja V, Chugh KS. Spectrum of hospital-acquired acute renal failure in the developing countries: Chandigarh study. Q J Med. 1992;84:497-505.

11 El-Lozi M, Akash N, Gneimat M, Smadi I, Nimri M, Hadidi M. Hospital acquired acute renal failure. Saudi J Kidney Dis Transpl. 1996;7(4):378-82.

12 Dlamini TAL, Heering PJ, Chivese T, Rayner B. A prospective study of the demographics, management and outcome of patients with acute kidney injury in Cape Town, South Africa. PLoS One. 2017;12(6):e0177460.

13 Goswami S, Pahwa N, Vohra R, Raju BM. Clinical spectrum of hospital-acquired acute kidney injury: a prospective study from central India. Saudi J Kidney Dis Transpl. 2018; 29:946-55.

14 Hou SH, Bushinsky DA, Wish JB, Cohen JJ, Harrington JT. Hospital-acquired renal insufficiency: a prospective study. Am J Med. 1983;74(2):243-8.

15 Nash K, Hafeez A, Hou S. Hospital-acquired renal insufficiency. Am J Kidney Dis. 2002; 39(5):930-6.
16 Welch HK, Kellum JA, Kane-Gill SL. Drugassociated acute kidney injury identified in the United States food and drug administration adverse event reporting system database. Pharmacotherapy. 2018;38(8):785-93.

17 Hosohata K, Inada A, Oyama S, Furushima D, Yamada H, Iwanaga K. Surveillance of drugs that most frequently induce acute kidney injury: a pharmacovigilance approach. J Clin Pharm Ther. 2019;44(1):49-53.

18 Zhou Y, Liu X, Ma L, Chen C, Yang T, Zhang $\mathrm{X}$, et al. Status analysis of clinical application and acute renal injury monitoring of vancomycin in some areas of China. Chin J Clin Pharmacol. 2019;35:82-6.

19 Pan K, Ma L, Xiang Q, Li X, Li H, Zhou Y, et al. Vancomycin-associated acute kidney injury: a cross-sectional study from a single center in China. PLoS One. 2017;12(4):e0175688.

20 Sun L, Tian S, Ma L, Zhao L, Zhou Y, Cui Y, et al. Comprehensive assessment system of vancomycins' rational use. Chin J Clin Pharmacol. 2014;30:143-5.

21 Nochaiwong S, Ruengorn C, Awiphan R, Koyratkoson K, Chaisai C, Noppakun K, et al. The association between proton pump inhibitor use and the risk of adverse kidney outcomes: a systematic review and meta-analysis. Nephrol Dial Transplant. 2018;33(2):331-42.

22 Qiu T, Zhou J, Zhang C. Acid-suppressive drugs and risk of kidney disease: a systematic review and meta-analysis. J Gastroenterol Hepatol. 2018;33(9):1566-73.
23 Muriithi AK, Leung N, Valeri AM, Cornell LD, Sethi S, Fidler ME, et al. Clinical characteristics, causes and outcomes of acute interstitial nephritis in the elderly. Kidney Int. 2015;87(2):458-64.

24 Muriithi AK, Leung N, Valeri AM, Cornell LD, Sethi S, Fidler ME, et al. Biopsy-proven acute interstitial nephritis: 1993-2011: a case series. Am J Kidney Dis. 2014;64:558-66.

25 Goldstein SL. Automated/integrated realtime clinical decision support in acute kidney injury. Curr Opin Crit Care. 2015;21(6):4859.

26 Osman M, Shigidi M, Ahmed H, Abdelrahman I, Karrar W, Elhassan E, et al. Pattern and outcome of acute kidney injury among Sudanese adults admitted to a tertiary level hospital: a retrospective cohort study. Pan Afr Med J. 2017;28:90-6.

27 Kohli HS, Bhat A, Jairam A, Aravindan AN, Sud K, Jha V, et al. Predictors of mortality in acute renal failure in a developing country: a prospective study. Ren Fail. 2007;29(4):4639.

28 Fang Y, Ding X, Zhong Y, Zou J, Teng J, Tang $\mathrm{Y}$, et al. Acute kidney injury in a Chinese hospitalized population. Blood Purif. 2010;30(2): $120-6$.

29 Wiedermann CJ, Wiedermann W, Joannidis M. Hypoalbuminemia and acute kidney injury: a meta-analysis of observational clinical studies. Intensive Care Med. 2010;36(10): 1657-65. 